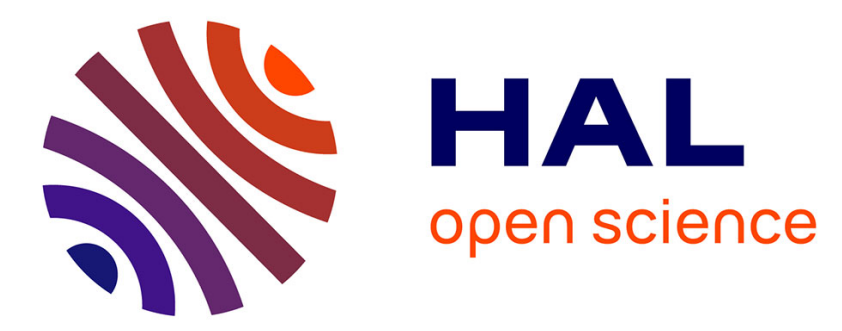

\title{
Prévision de la réponse à la trempe des aciers en fonction de l'efficacité des fluides de trempe
}

\author{
G. Schanne, G. Kellen, C. Weider
}

\section{To cite this version:}

G. Schanne, G. Kellen, C. Weider. Prévision de la réponse à la trempe des aciers en fonction de l'efficacité des fluides de trempe. Journal de Physique IV Proceedings, 1993, 03 (C7), pp.C7-1223-C71228. 10.1051/jp4:19937188 . jpa-00251820

\section{HAL Id: jpa-00251820 https://hal.science/jpa-00251820}

Submitted on 1 Jan 1993

HAL is a multi-disciplinary open access archive for the deposit and dissemination of scientific research documents, whether they are published or not. The documents may come from teaching and research institutions in France or abroad, or from public or private research centers.
L'archive ouverte pluridisciplinaire HAL, est destinée au dépôt et à la diffusion de documents scientifiques de niveau recherche, publiés ou non, émanant des établissements d'enseignement et de recherche français ou étrangers, des laboratoires publics ou privés. 


\title{
Prévision de la réponse à la trempe des aciers en fonction de l'efficacité des fluides de trempe
}

\author{
G. SCHANNE, G. KELLEN et C. WEIDER
}

UNIMETAL Recherche, BP. 140, 57360 Amneville, France

\begin{abstract}
Quenching makes it possible to obtain, on previously austenitized steel parts, special structures and mechanical characteristics by making faster cooling than in calm air. To obtain this cooling, the austenitized part is plunged into a stirred fluid maintained at low temperature. After having recalled the methods making it possible to quantify the effectiveness of the quenching fluid and to foresee the aptitude of a steel grade for quenching (or hardenability), we will specify the method developed by UNIMETAL RECHERCHE :

- to control, on an instrumented furnace, the quenching parameters (mainly, fluid characteristics)

- to determine the cooling curves inside cylindrical steel test bars ( $\Phi$ 10 to $60 \mathrm{~mm}$ )

- to measure cooling speeds (Vr) at specific points on the incident curves

- to establish of the relationship between $\mathrm{Vr}$ and the diameter $\Phi$

- to enter these relations into the PREVER UNIMETAL model. This makes it possible to foresee the results of conventional tests defining hardenability (JOMINY test, $\mathrm{U}$ curves)
\end{abstract}

This model, operational in 1993, makes it possible to avoid long and costly real tests. 


\section{1 - INTRODUCTION}

La trempe est le traitement thermique permettant d'obtenir sur des pièces en acier, austénitisées au préalable, et ceci grâce à un refroidissement accéléré, des caractéristiques structurales et mécaniques déterminées. Le refroidissement accéléré s'obtient généralement en introduisant rapidement le métal austénitisé dans un fluide agité et maintenu à basse température.

\section{2 - CARACTERISTIQUES PROPRES A LA NUANCE}

Deux essais principaux permettent de déterminer l'aptitude de la nuance d'acier à être trempée, c'est-à-dire la trempabilité. Le principe de ces essais est précisé dans les schémas ci-dessous :

\section{COURBE EN U}

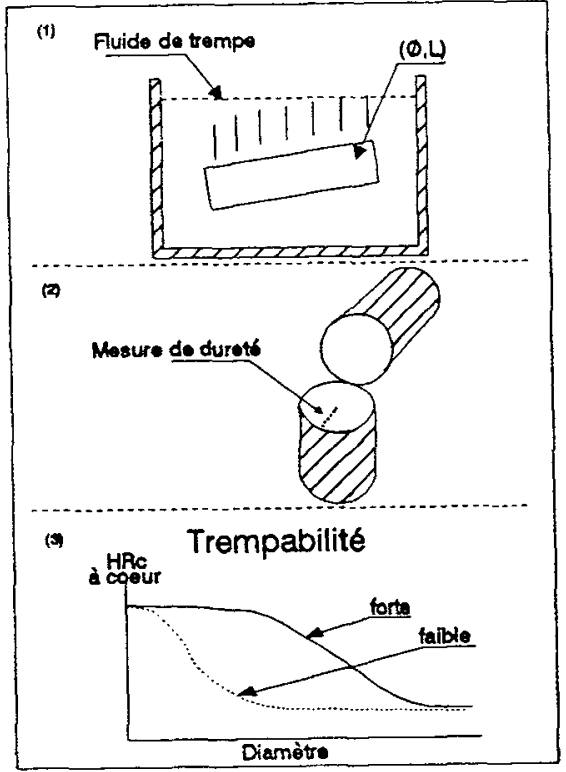

ESSAI JOMINY

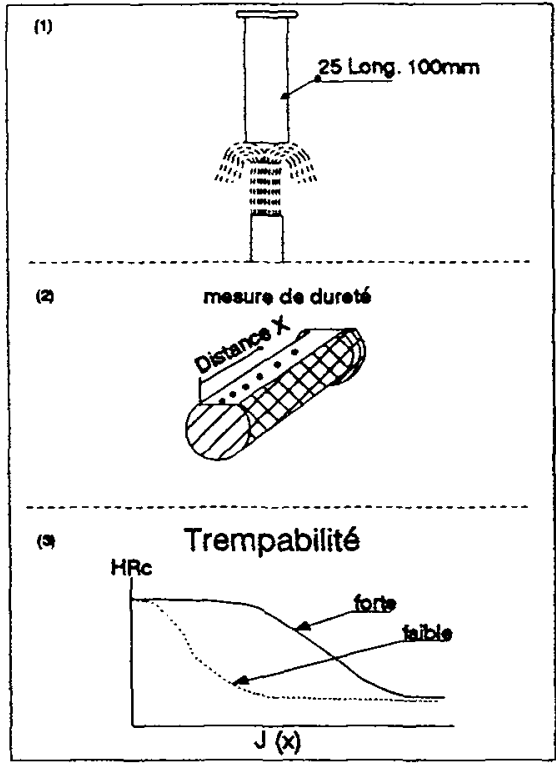

- Essai Jominy : après austénitisation, la pièce est refroidie par un jet d'eau perpendiculaire à la face inférieure (1). On mesure la dureté sur un méplat usiné longitudinalement (2) et on reporte la valeur de dureté en fonction de la distance de l'extrémitê trempée (3) (essai défini par la norme NFA 04-303)

- Courbe en U : après austénitisation, l'échantillon cylindrique est trempé dans un fluide déterminé (1). On mesure l'évolution de dureté sur un diamètre du plan médian (2) et on reporte la dureté à coeur en fonction du diamètre (3) 


\section{3 - CARACTERISTIQUES DU FLUIDE}

Deux méthodes principales sont utilisées pour quantifier l'efficacité d'un fluide : le paramètre de sévéritê $\mathrm{H}$ et la courbe de drasticité :

- le paramètre $H$ est défini par la relation $H=\frac{h}{2} \lambda$ (suivant GROSSMANN) où $h$ est le coefficient d'échange moyen et $\lambda$ la conductivité thermique moyenne. H s'exprime en mm- mais n'est pas d'un emploi facile car le coefficient d'échange dépend de la forme et dimension de la pièce traitée

- la courbe de drasticité, définie par la norme NFT 60178, qui consiste à mesurer la loi de refroidissement à coeur d'une éprouvette cylindrique en argent et à tracer la courbe en refroidissement continu et sa dérivée

Ces deux méthodes n'étant pas utilisables dans le cas du modèle PREVERUNIMETAL, nous avons été amenés à utiliser une méthode nouvelle.

\section{4 - METHODE MISE AU POINT A UNIMETAL RECHERCHE}

La méthode consiste à :

- utiliser un four instrumenté (pl. 1)

- mesurer les lois de refroidissement à coeur d'échantillons cylindriques de diamètres variables, pour les divers fluides (pl. 2).

- l'examen des courbes de drasticité d'un fluide de trempe, l'eau par exemple, montre qu'on peut y définir deux grands domaines :

* celui des températures élevêes $\left(\approx 800 \mathrm{a} \approx 600^{\circ} \mathrm{C}\right.$ ) où se détermine la structure de trempe : la vitesse doit être la plus grande possible pour obtenir les duretés optimales

* celui des températures moyennes $\left(\approx 500\right.$ à $\left.\approx 300{ }^{\circ} \mathrm{C}\right)$ oũ se forment d'éventuelles tapures de trempe dues aux contraintes élevées liées à un refroidissement rapide. La vitesse doit être faible dans ce domaine, ce qui explique que pour les nuances les plus trempantes let les plus fragiles), il soit nécessaire d'utiliser de l'huile comme fluide de trempe.

Nous mesurons donc pour les divers fluides les vitesses de refroidissement dans ces deux domaines, et ceci en fonction du diamètre.

Pour quantifier la dépendance entre vitesses de refroidissement et diamètres, nous déterminons des relations du type : $\log V r=A-B \log \Phi$ pour des intervalles donnés de diamètres.

- les planches 3 et 4 indiquent les résultats obtenus pour divers fluides de trempe, respectivement dans les domaines supérieurs $\left(\approx 700{ }^{\circ} \mathrm{C}\right)$ et inférieurs $\left(\approx 350^{\circ} \mathrm{C}\right)$

\section{5 - INTEGRATION DANS LE MODELE PREVER-UNIMETAL}

La planche 5 indique le principe général de fonctionnement du modèle de départ (PREVERT, mis au point par la Société CREUSOT LOIRE INDUSTRIE). Nous avons adapté ce modèle aux nuances d'UNIMETAL et développé un modèle trempabilité avec : 
- entrée de l'analyse : et tri automatique entre trois familles d'acier pour traitement thermique ( $\mathrm{XC}$, acier au bore, aciers faiblement alliés)

- choix entre essai Jominy, ou

- courbe en U avec sélection du fluide

- sortie des résultats sur un écran ou par voie graphique

Les planches 6,7 et 8 illustrent des exemples d'utilisation du modèle.

\section{6 - CONCLUSION}

Pour trois familles d'aciers de traitement thermique $(X C$, acier au bore, acier faiblement allié, voir tableau ci-dessous), le modèle PREVERUNIMETAL permet de prévoir avec une bonne précision la trempabilité des aciers en donnant des résultats calculés pour l'essai Jominy et la courbe en $U$.

\begin{tabular}{|l|c|c|c|c|c|c|c|c|}
\hline \multicolumn{2}{|c|}{} & \multicolumn{2}{c|}{ Mn } & \multicolumn{2}{c|}{ Cr } & \multicolumn{2}{c|}{ Si } \\
\hline & mini & MAXI & mini & MAXI & mini & MAXI & mini & MAXI \\
\hline XC & 220 & 600 & 650 & 1015 & 35 & 400 & 35 & 340 \\
\hline Faibl. Alliés & 200 & 500 & 740 & 1360 & 160 & 1150 & 220 & 380 \\
\hline Bore & 150 & 460 & 510 & 1300 & 100 & 380 & 60 & 280 \\
\hline
\end{tabular}




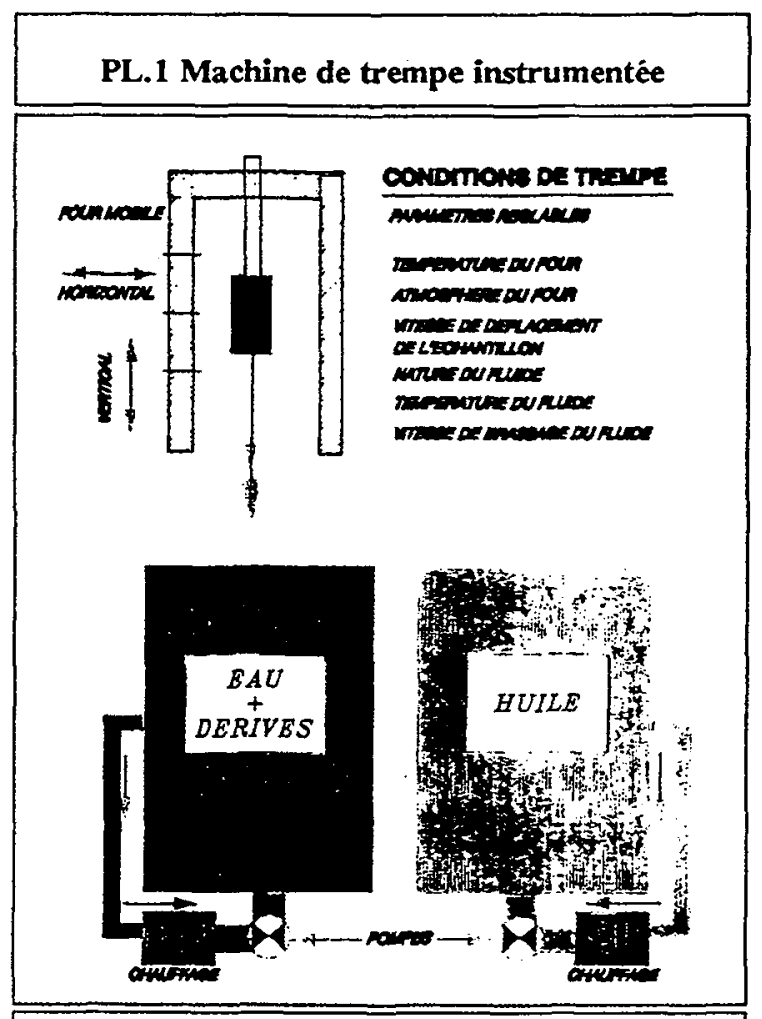

PL.3 Vitesse de refroidissement à $700^{\circ} \mathrm{C}$

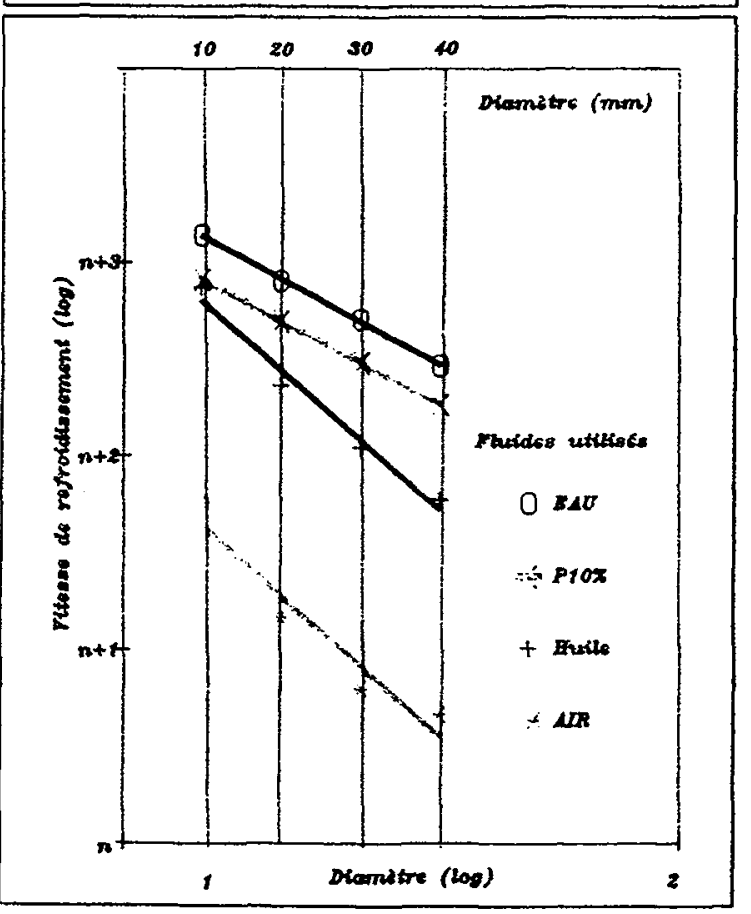

PL.2 Lois de refroidissement de l'eau

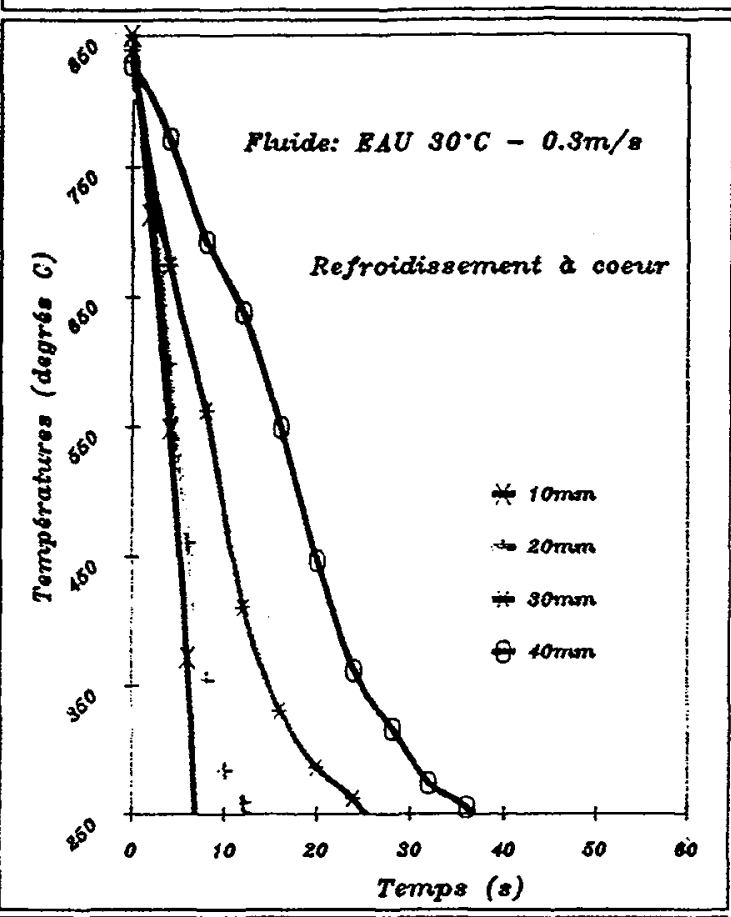

PL.4 Vitesse de refroidissement à $350^{\circ} \mathrm{C}$

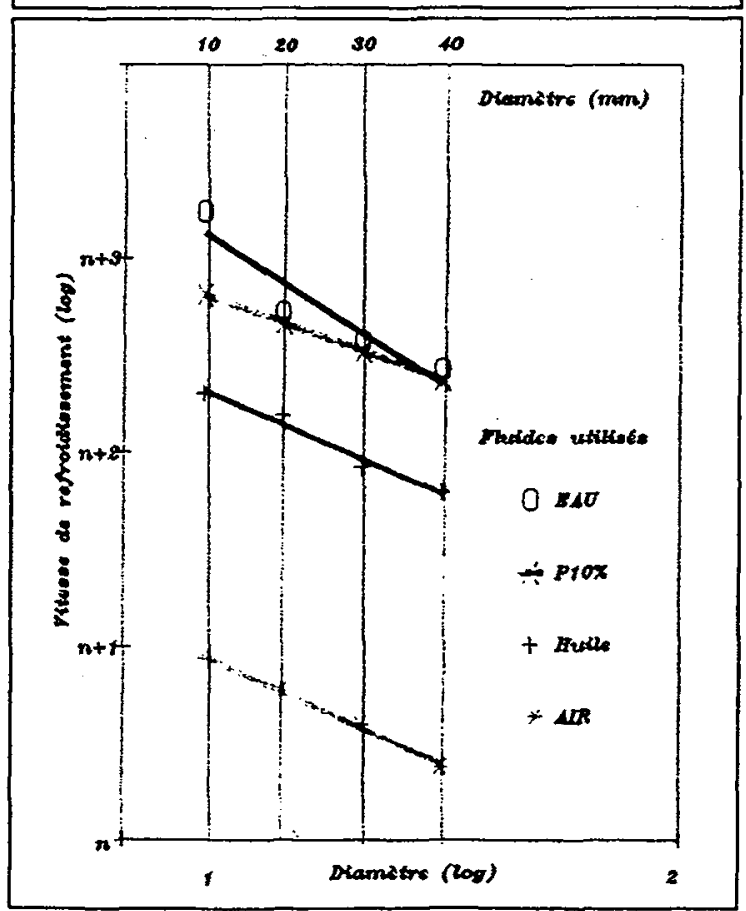


PL.5 Principe du modèle PREVERT

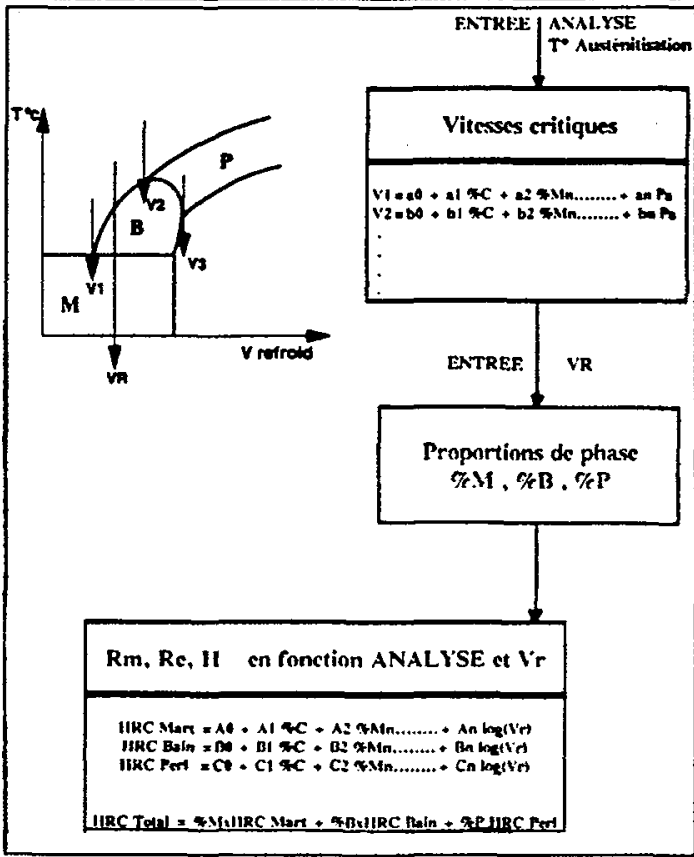

PL.7 Courbe en U: exemples d'utilisation

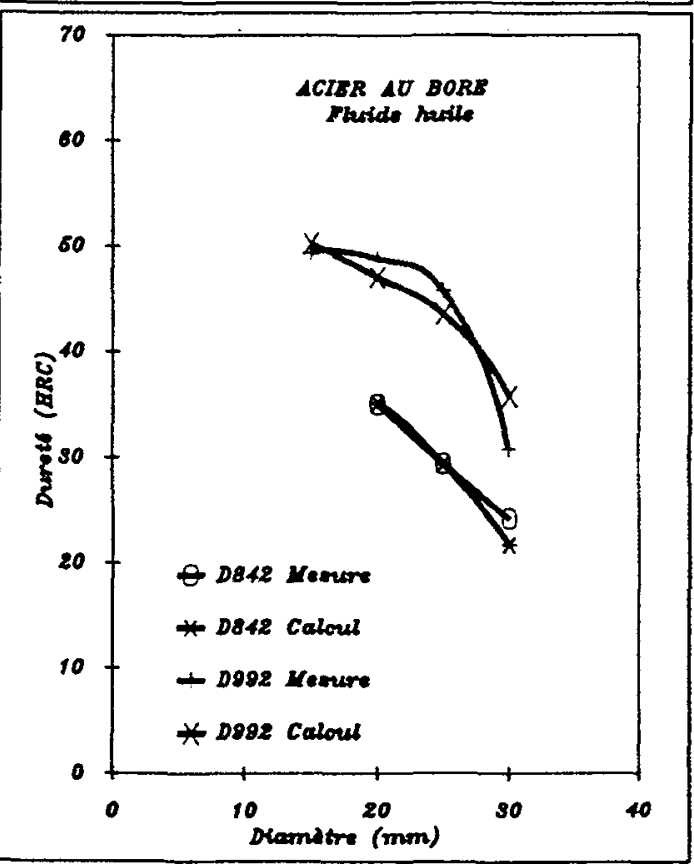

\section{PL.6 Validation de l'essai JOMINY}

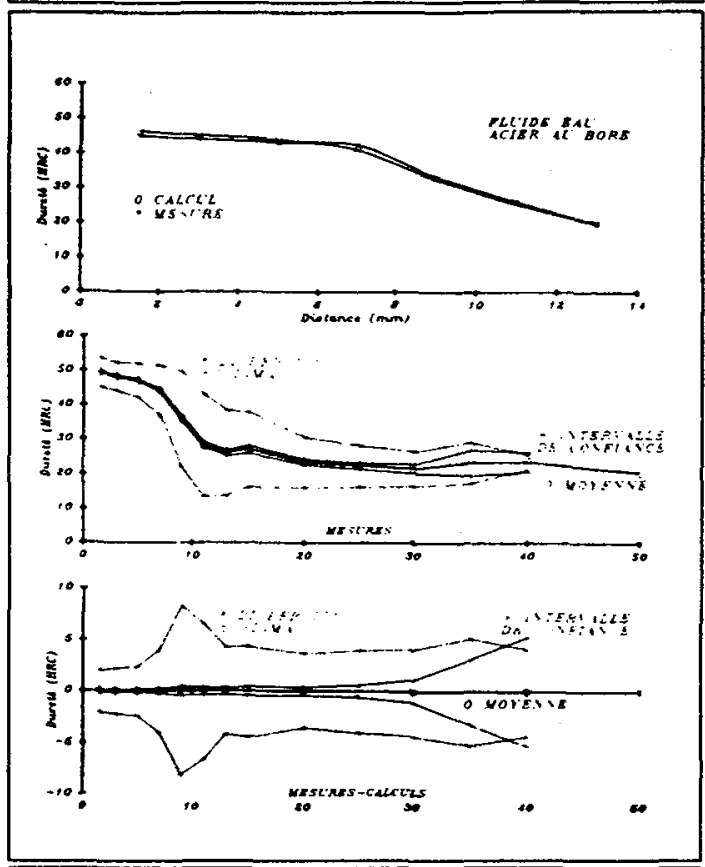

PL.8 Courbe en U: exemples d'utilisation

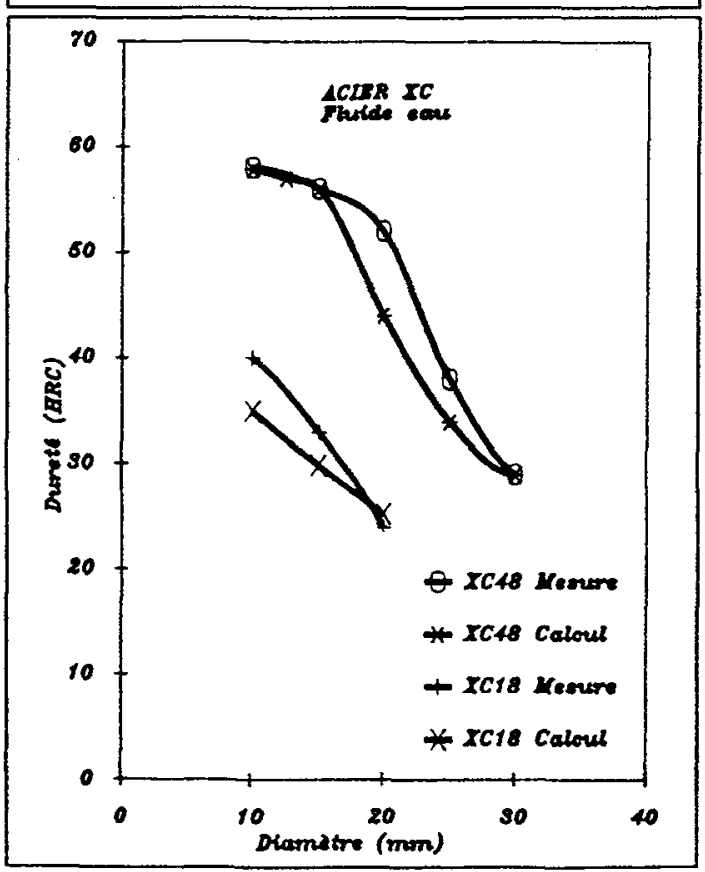

